\title{
Topical ivermectin in the treatment of pediculosis capitis
}

\section{Luma Husni, Makram Al-Waiz}

\author{
Baghdad, Medical City, Baghdad Training Centre, Baghdad, Iraq
}

Corresponding author: Dr. Luma Husni, E-mail: sizerluma@yahoo.com

\begin{abstract}
Background: Head lice infestation is considered a prominent issue because of its worldwide distribution, especially among children. Millions of children are infested with pediculosis capitis every year. The increasing rate of lice infestation has been reported even in the developed countries due to the resistance to known pediculicides. Besides, the louse is a vector for serious diseases, such as epidemic typhus fever. Objective: The aim of this work was the search for a new cheap and effective drug for pediculosis capitis. $2 \%$ ivermectin solution, a promising novel drug used for endoparasite and ectoparasite infections, was used in this study. Materials and Methods: This was a clinical trial study. The in vitro study was carried out in a period of five months. Samples of nits and lice were collected from female patients of the Department of Dermatology and Venereology, Baghdad Teaching Hospital. The study was conducted during a period from October 2002 through November 2003. One hundred fifty children from four primary schools in Hayy Al-A'amel, Baghdad, participated in this part of the study. The preparation was applied to the children by the author. One hundred children were tested with ivermectin solution and fifty children with rectified spirit. The first part of the study involved a parasitological evaluation (in vitro study) of pediculicidal and ovicidal activity, in which $4 \%$ ivermectin solution was discontinued because of contact dermatitis. Therefore, $2 \%$ ivermectin solution was used instead. Pediculicidal and ovicidal activity was examined half an hour afterward. The second part of the study involved a clinical evaluation (in situ study) of 2\% ivermectin solution for half an hour. Results: Topical 2\% ivermectin solution in rectified spirit is a good pediculicidal (100\%) and a good ovicidal (88\%) drug with good therapeutic efficacy (82\% after the first application, $90 \%$ after the second application) against pediculosis capitis. Conclusion: The study described a novel topical preparation for pediculosis capitis, which was proven effective and safe.
\end{abstract}

Key words: Ivermectin; Pediculosis capitis; Iraq; Topical solution

\section{INTRODUCTION}

Population-based studies in European countries show a highly divergent prevalence of pediculosis capitis, ranging from $1 \%$ to $20 \%$ [1]. With an increasing rate of treatment failure, it is worthwhile to consider the issues of misdiagnosis, lack of adherence, inadequate treatment, reinfestation, lack of ovicidal or residual killing properties of the pediculicide, and/or resistance of lice to the pediculicide [2]. Resistance to topical pediculicides is an emerging concern in most parts of the world [3]. The problem of resistance is directly related to the frequency of its use $[4,5]$ in genotyping, demonstrating that head lice exhibit resistance to compounds such as
DDT, the pyrethrins, and the pyrethroids [6]. Numerous treatment options have been used to control the spread of pediculosis capitis with various drawbacks. For instance, $1 \%$ lindane exhibits central nervous system toxicity and may cause severe seizures in children [7]. 5\% benzyl alcohol was the first non-neurotoxic pediculicide, and pruritus, erythema, pyoderma, and ocular irritation are its usual side effects. It is also non-ovicidal [8]. The percentage of patients who were louse-free after treatment with a product containing tea tree oil and lavender oil and a head lice suffocation product was higher than with a product containing pyrethrins and piperonyl butoxide [9]. Eucalyptus oils have been used against permethrin-resistant pediculosis [10]. 
Grapefruit juice [11], coconut, and anise spray have been found to be more effective than permethrin in pediculosis capitis, thereby demonstrating clinical resistance [12].

In head lice infestations that are difficult to treat, oral ivermectin may prove more effective [13] or as effective as topical $0.5 \%$ malathion lotion [14]. Oral ivermectin demonstrates high efficacy and tolerability in the treatment of pediculosis capitis. A significant number of children required a second dose to ensure complete eradication [15]. A single oral dose of ivermectin $400 \mu \mathrm{g} /$ $\mathrm{kg}$ repeated throughout seven days has been shown to be more effective than $0.5 \%$ malathion lotion [13]. It has also been used successfully in school children [16]. In head lice infestations that are difficult to treat, oral ivermectin given twice every seven days had superior efficacy when compared with topical $0.5 \%$ malathion lotion [17]. After a single dose, complete healing was achieved in $77.5 \%$ and $87.5 \%$ of ivermectin and malathion groups, respectively. No major adverse effects were observed in either group. Oral ivermectin is a promising and effective approach for the treatment of head lice and might be an ideal substitute for conventional pediculicides [14]. Community-based treatment with oral ivermectin in susceptible, and poor sections of the society has given us promising results [18].

\section{MATERIALS AND METHODS}

In vitro parasitological evaluation: The activity of the test preparation was evaluated on the Pediculus capitis in ovicidal and pediculicidal tests. Samples of nits and lice were collected from female patients of the Department of Dermatology and Venereology, Baghdad Teaching Hospital. The study was conducted during a period from September 2002 through November 2003. 2\% ivermectin solution $1 \mathrm{gm} / 50 \mathrm{cc}$ rectified spirit was used and tested by parasitological evaluation (in vitro study). A control test was included as part of the parasitological evaluation as well. Rectified spirit was the control preparation. Ovicidal tests of each type of test and control preparation were tested half an hour after washing in tap water.

Pediculicidal test: lice and nymphs were collected from the patients' heads with a fine-tooth comb, gently transferred with forceps onto a clean Petri dish, closed with self-adhesive tape, and identified. Lice were examined microscopically. Lice that were dead, damaged, or less than fully mobile were discarded. Fully mobile (active) lice were transferred to another clean Petri dish. A hundred fully mobile lice were exposed to test preparations by immersion at room temperature. The number of live and dead lice was recorded. After three minutes, the lice were washed in tap water, then microscopically examined. A louse was considered dead if it lacked signs of internal and external movement. Fifty fully mobile lice were exposed to rectified spirit half an hour after washing, then microscopically examined. The percentage of dead lice represented the pediculicidal activity of the test preparation.

\section{Ovicidal Test}

Hair shafts with attached viable ova (nits) were removed with forceps and scissors, transferred onto a sterile Petri dish, closed with self-adhesive tape, and identified. An ovum was judged viable if it was plump, had an intact operculum and ideal eyespot, and was yellow or creamy white. Dark tan to brown or black ova with shriveled or shrunken shells were judged nonviable. An ovum that had the operculum popped open and a translucent shell without a brown residue was considered hatched. Nits were examined with a binocular microscope. Empty (hatched) or possibly dead (nonviable) nits were excluded from the ovicidal test, whereas the plump (viable) nits were transferred to another clean Petri dish. The viable ova, firmly fixed to their support (hairs), were exposed to the test preparation by immersion for half an hour at room temperature. Afterward, the nits were washed three times in tap water. Nits were allowed to dry at room temperature and incubated again in similar conditions at room temperature for two weeks for hatching. Next, unhatched nits were counted under the microscope. The percentage of unhatched nits represented the ovicidal activity of the test preparation. Then, the same was done but with the control preparation after the completion of the parasitological evaluation.

\section{In Situ Study}

Ivermectin solution was tested for its therapeutic efficacy on primary school children infested with pediculosis capitis. One hundred fifty children from four primary schools in Hayy Al-A'amel, Baghdad, participated in this part of the study.

The children were in grades one to six. The preparation was applied to the children by the author. One hundred children were tested with ivermectin solution and fifty children with rectified spirit. 
To be eligible for participation, each patient had to have a diagnostically active head lice infestation confirmed by direct visual identification of live adult lice or nymphs. The presence of nits on the hair shafts was insufficient to qualify a child for inclusion in the study. A child was excluded if there were any other dermatological conditions present or if a pediculicide was used within two weeks of the initial evaluation.

The parents agreed not to wash the hair of their children for half an hour of solution application and not to use pediculicides, medicated shampoos, or lotions, other than ordinary shampoo, during the study. During the first visit, a visual estimation of live adult louse and nymph populations in the children's hair was conducted and the test preparation was applied to the dry scalp in amounts sufficient to thoroughly wet the hair and skin of the infested areas. An amount of $25-50 \mathrm{~mL}$ was sufficient to wet the hair and scalp, but extremely long and thick hair sometimes required larger amounts. During the application of the test preparation, the children were instructed to protect their eyes from the preparation by holding a folded towel against their forehead. The hair was covered with a cup for half an hour, then washed.

The therapeutic index (i.e., eradication of live lice and nymphs) was evaluated on days seven and fourteen following the treatment. The children were examined for a minimum of five minutes for the presence of one or more live adult lice or nymphs on either evaluation. The first application of the preparation was given at the first visit, and the second application was given at the second visit (after one week) with the same procedure as on the first application. The parents of the children were instructed to boil or steam sheets, pillowcases, and other formats for about 15-20 minutes.

A clinical evaluation was performed 30-60 minutes following day fourteen. The children were examined for three dermal signs (edema, erythema, and rash) and five dermal symptoms (pruritus, burning, stinging, numbness, and tingling).

\section{Ethics Statement}

All authors hereby declare that the study has been approved by the scientific committee of the Scientific Council of Dermatology and have, therefore, been performed in accordance with the ethical standards defined by the Iraqi Board of Dermatology and Venereology.

\section{RESULTS}

A total of 150 lice were included in the study. A hundred were tested with ivermectin and fifty with rectified spirit.

The following pediculicidal activity was observed:

1. $2 \%$ ivermectin solution

After exposure for three minutes, all of the 100 ova were dead. The pediculicidal activity of the preparation was $100 \%$.

2. Rectified spirit

After half an hour, 7 ova out of the 50 were dead. The pediculicidal activity was $14 \%$.

\section{Ovicidal Test Results}

A total of 150 eggs were included in the study.

1. Out of the 100 eggs, 88 eggs were dead. The ovicidal activity of the ivermectin solution was $88 \%$ after half an hour.

2. Out of the 50 eggs, 6 eggs were dead. The ovicidal activity of the rectified spirit was $12 \%$ after half an hour.

The lice were found to be able to survive for 2-4 days at $30^{\circ} \mathrm{C}$ without food (away from the human host).

\section{In Situ Study Results}

Hundred fifty primary school children infested with active pediculosis capitis were included in the study. One hundred of them were treated with $2 \%$ ivermectin solution at the first and second visit and fifty with a control preparation (rectified spirit). All children were female, and their age ranged from 6 to 12 years with a mean of 9 years.

Eighty-two children had no live lice at the first examination (one week after the first application of the test preparation). The therapeutic efficacy of the test preparation after one application was $82 \%$, and 90\% after two applications.

While out of the 50 children infested with pediculosis capitis treated with rectified spirit applied for half an hour before washing, 47 children had live lice (adults or nymphs) at the first examination (one week after the first application), and 43 children had live lice (adults or nymphs) at the second examination (one week after the second application). Therefore, the therapeutic efficacy 
of the control preparation (rectified spirit) was 6\% after the first application and $14 \%$ after the second application.

A burning sensation was reported in twelve children (12\%) 15-30 minutes after the application of the test solution ( $2 \%$ ivermectin solution). No other dermal symptoms or signs were observed after the application of either preparation.

\section{DISCUSSION}

Ivermectin was chosen because of its role in the treatment of ectoparasitic infestations and its relative lack of toxicity as compared with other modalities by recent studies and, because of the increasing number of treatment failures, possibly due to the development of resistance, a change in treatment is to be considered.

Ivermectin is a new drug for the treatment of ectoparasites. To determine its effect on pediculosis capitis, in this study, we applied it as a topical 2\% solution in rectified spirit and found that the in vitro pediculicidal activity of the ivermectin solution after three minutes was exceptionally good $(100 \%)$ and that its ovicidal activity was good as well (88\%).

After the first application of $2 \%$ ivermectin solution for half an hour, the therapeutic efficacy (percentage of dead lice and ova) was very good (82\%) and, after the second application, it was still very good $(90 \%)$.

A burning sensation was reported in twelve children 30 minutes after the application. These results support that topical ivermectin solutions produce a low frequency of side effects, whereas, with the use of lindane, toxicity has been reported [7].

The result of the study indicates that a $2 \%$ ivermectin solution is a good pediculicidal and ovicidal drug that appears to be a suitable alternative, especially considering the reported worldwide spread of resistance to both pyrethroids and malathion.

Therefore, $2 \%$ ivermectin solution is a good and cheap pediculicide with a low frequency of side effects. Its main advantages are low adverse reactions and rapid insecticide action, which requires a short contact time and makes it suitable for home treatment.

The study suggests that topical ivermectin may be a promising treatment for head lice and that a second dose on day seven might be appropriate.
$2 \%$ ivermectin solution was found to have good ovicidal activity (88\%) and, if the time of exposure is increased, the ovicidal activity may increase; therefore, a second application is of extreme importance.

In the study, the lice were able to survive for 2-4 days without food (away from the human host).

Causes of treatment failure: Active lice, adults and nymphs, in parasitological evaluation, exposed to the test preparation under optimal conditions with complete exposure of all lice to the preparation in a Petri dish, while the application of the preparation on the hair of the children may not be in close contact with all the lice. Some nits may need more than one week to hatch. Therefore, the nits will hatch on the second application, and this may lead to failure in treatment and reinfestation since all failed cases in our study had live lice. Most of them were small (nymphs) but several were of a mature size (adults), suggesting that these children had been reinfested.

\section{CONCLUSION}

This is the first Iraqi study on the use of topical ivermectin for pediculosis capitis.

Topical 2\% ivermectin solution in rectified spirit is a good pediculicidal (100\%) and a good ovicidal $(88 \%)$ drug for the in vitro treatment of pediculosis capitis. However, a second application seems to be necessary because of its incomplete ovicidal activity.

It is deemed safe and has no or few side effects.

It is a good pediculicide drug, with $82 \%$ therapeutic efficacy on the first application and 90\% therapeutic efficacy on the second application, and a good choice for a primary treatment of pediculosis capitis and resistant cases.

At the time of the study, there had been few similar studies, which shows that there is a need for more clarification on Iraqi patients because of the geographical diversity of resistant cases.

\section{Recommendations}

1. Further studies regarding ivermectin solutions for longer periods of exposure. 
2. Further comparative studies of ivermectin solutions with other pediculicides.

3. There is an urgent need for monitoring the development of resistance through official control of sales and prescriptions.

4. New products such as topical ivermectin are required and, once introduced, careful control of their use would be of benefit.

5. Control of head lice can be attempted by head shaving and wet combing.

6. We recommend the use of a fine-toothed louse comb in addition to treatment for its role in:

a. The prevention of lice infestation;

b. Anti-louse treatment methods as an accessory tool;

c. The removal of nits.

Mass family treatment is necessary.

\section{ACKNOWLEDGMENTS}

We are grateful to Dr. Abdul-Rahman Al-Tae and Dr. Raad Al-Sady for their encouragement and help in providing us with necessary observations.

\section{Statement of Human and Animal Rights}

All the procedures followed were in accordance with the ethical standards of the responsible committee on human experimentation (institutional and national) and with the 2008 revision of the Declaration of Helsinki of 1975.

\section{Statement of Informed Consent}

As per the national standard, written consent has been collected from the patient or a parent of the patient and preserved by the author(s).

\section{REFERENCES}

1. Feldmeier H. Pediculosis capitis: New insights into epidemiology, diagnosis and treatment. Eur J Clin Microbiol Infect Dis. 2012;31:2105-10.

2. Frankowski BL, Bocchini JA, Jr. Council on School Health and Committee on Infectious Diseases. Head lice. Pediatrics. 2010;126:392-403.

3. Marcoux D, Palma KG, Kaul N, Hodgdon H, Van Geest A, Previte DJ, et al. Pyrethroid pediculicide resistance of head lice in Canada evaluated by serial invasive signal amplification reaction.
J Cutan Med Surg. 2010;14:115-8.

4. Bialek R, Zelck UE, Fölster-Holst R. Permethrin treatment of head lice with knockdown resistance-like gene. N Engl J Med. 2011;364:386-7.

5. Lee SH, Gao J-R, Yoon KS, Mumcuoglu KY, Taplin D, Edman JD, et al. Sodium channel mutations associated with knockdown resistance in the human head louse, Pediculus capitis (De Geer). Pestic Biochem Physiol. 2003;75:79-91.

6. Hodgdon HE, Yoon KS, Previte DJ, Kim HJ, Aboelghar GE, Lee SH, et al. Determination of knockdown resistance allele frequencies in global human head louse populations using the serial invasive signal amplification reaction. Pest Manag Sci. 2010;66:1031-40.

7. Nolan K, Kamrath J, Levitt J. Lindane Toxicity: A comprehensive review of the medical literature. Pediatr Dermatol. 2012;29:141-6.

8. Meinking TL, Villar ME, Vicaria M, Eyerdam DH, Paquet D, MertzRivera K, et al. The clinical trials supporting benzyl alcohol lotion 5\% [Ulesfia]: A safe and effective topical treatment for head lice [pediculosis humanus capitis]. Pediatr Dermatol. 2010;27:19-24.

9. Barker SC, Altman PM. A randomized, assessor blind, parallel-group comparative efficacy trial of three products for the treatment of head lice in children-melaleuca oil and lavender oil, pyrethrins and piperonyl butoxide, and a "suffocation" product. BMC Dermatol. 2010;10:6.

10. Toloza AC, Lucía A, Zerba E, Masuh H, Picollo MI. Eucalyptus essential oil toxicity against permethrin-resistant Pediculus humanus capitis [Phthiraptera: Pediculidae]. Parasitol Res. 2010;106:409-14.

11. Abdel-Ghaffar F, Semmler M, Al-Rasheid K, Klimpel S, Mehlhorn H. Efficacy of a grapefruit extract on head lice: A clinical trial. Parasitol Res. 2010;106:445-9.

12. Burgess IF, Brunton ER, Burgess NA. Clinical trial showing superiority of a coconut and anise spray over permethrin $0.43 \%$ lotion for head louse infestation, ISRCTN96469780. Eur J Pediatr. 2010;169:55-62.

13. Chosidow O, Giraudeau B, Cottrell J, Izri A, Hofmann R, Mann SG, et al. Oral ivermectin versus malathion lotion for difficult-to-treat head lice. N Engl J Med. 2010;362:896-905.

14. Nofal A. Oral ivermectin for head lice: A comparison with $0.5 \%$ topical malathion lotion. J Dtsch Dermatol Ges. 2010;8:985-8.

15. Ameen M, Arenas R, Villanueva-Reyes J, Ruiz-Esmenjaud J, Millar D, Domínguez-Dueñas F, et al. Oral ivermectin for treatment of pediculosis capitis. Pediatr Infect Dis J. 2010;29:991-3.

16. Currie MJ, Reynolds GJ, Glasgow NJ, Bowden FJ. A pilot study of the use of oral ivermectin to treat head lice in primary school students in Australia. Pediatr Dermatol. 2010;27:595-9.

17. Chosidow O, Giraudeau B, Cottrell J, Izri A, Hofmann R, Mann SG, Burgess I. Ivermectin versus malathion lotion for difficult-to-treat head lice. N Engl J Med. 2010;362:896-905.

18. Pilger D, Heukelbach J, Khakban A, Oliveira FA, Fengler G, Feldmeier H. Household-wide ivermectin treatment for head lice in an impoverished community: Randomized observer-blinded controlled trial. Bull World Health Organ. 2010;88:90-6.

Copyright by Luma Husni, et al. This is an open-access article distributed under the terms of the Creative Commons Attribution License, which permits unrestricted use, distribution, and reproduction in any medium, provided the original author and source are credited.

Source of Support: Nil, Conflict of Interest: None declared. 